Article

\title{
Comparison of Branched and Linear Perfluoropolyether Chains Functionalization on Hydrophobic, Morphological and Conductive Properties of Multi-Walled Carbon Nanotubes
}

\author{
Maurizio Sansotera ${ }^{1,2, *}$, Sadaf Talaeemashhadi ${ }^{1}$, Cristian Gambarotti ${ }^{1,2}$, Carlo Pirola ${ }^{3,4}$ (D), \\ Mariangela Longhi ${ }^{3}(\mathbb{D})$, Marco A. Ortenzi ${ }^{3}$, Walter Navarrini ${ }^{1,2}$ and Claudia L. Bianchi ${ }^{3,4, *}$ (i) \\ 1 Dipartimento di Chimica, Materiali e Ingegneria Chimica, Politecnico di Milano, via Mancinelli 7, \\ I-20131 Milano, Italy; sadaf.talaeemashhadi@chem.polimi.it (S.T.); cristian.gambarotti@polimi.it (C.G.); \\ walter.navarrini@polimi.it (W.N.) \\ 2 Consorzio Interuniversitario Nazionale per la Scienza e Tecnologia dei Materiali (UdR-PoliMi), \\ via G. Giusti, 9, 50121 Firenze, Italy \\ 3 Dipartimento di Chimica, Università degli Studi di Milano, via Golgi 19, I-20133 Milano, Italy; \\ carlo.pirola@unimi.it (C.P.); mariangela.longhi@unimi.it (M.L.); marco.ortenzi@unimi.it (M.A.O.) \\ 4 Consorzio Interuniversitario Nazionale per la Scienza e Tecnologia dei Materiali (UdR-UniMi), \\ via G. Giusti, 9, 50121 Firenze, Italy \\ * Correspondence: maurizio.sansotera@polimi.it (M.S.); claudia.bianchi@unimi.it (C.L.B.); \\ Tel.: +39-02-2399-4770 (M.S.); +39-02-5031-4253 (C.L.B.)
}

Received: 14 February 2018; Accepted: 15 March 2018; Published: 19 March 2018

\begin{abstract}
The functionalization of multi-walled carbon nanotubes (MW-CNTs) was obtained by generating reactive perfluoropolyether (PFPE) radicals that can covalently bond to MW-CNTs' surface. Branched and linear PFPE peroxides with equivalent molecular weights of 1275 and $1200 \mathrm{amu}$, respectively, have been thermally decomposed for the production of PFPE radicals. The functionalization with PFPE chains has changed the wettability of MW-CNTs, which switched their behavior from hydrophilic to super-hydrophobic. The low surface energy properties of PFPEs have been transferred to MW-CNTs surface and branched units with trifluoromethyl groups, $\mathrm{CF}_{3}$, have conferred higher hydrophobicity than linear units. Porosimetry discriminated the effects of PFPE functionalization on meso-porosity and macro-porosity. It has been observed that reactive sites located in MW-CNTs mesopores have been intensively functionalized by branched PFPE peroxide due to its low average molecular weight. Conductivity measurements at different applied pressures have showed that the covalent linkage of PFPE chains, branched as well as linear, weakly modified the electrical conductivity of MW-CNTs. The decomposed portions of PFPE residues, the PFPE chains bonded on carbon nanotubes, and the PFPE fluids obtained by homo-coupling side-reactions were evaluated by mass balances. PFPE-modified MW-CNTs have been characterized by X-ray photoelectron spectroscopy (XPS), scanning electron microscopy (SEM), thermogravimetric analysis (TGA), static contact angle (SCA), surface area, and porosity measurements.
\end{abstract}

Keywords: carbon nanotubes; functionalization; surface chemistry; superhydrophobicity

\section{Introduction}

Carbon nanotubes (CNTs) are chemically stable and mechanically resistant due to their rolled-up graphene planes with extensively delocalized $\pi$ electron systems [1]. However, the lack of solubility in any solvent and the difficulty in manipulation have imposed great limitations to the application of CNTs [2-9]. In order to improve their ability to disperse and to facilitate their manipulation, 
many researchers have attempted the functionalization of CNT surface $[10,11]$. Several sidewall derivatization methods were developed by using fluorine [12], carbenes [13], azomethine ylides [14], and/or organic radicals [15]. Among these approaches, CNTs fluorination has been identified as one of the most effective chemical methods to modify and control CNTs physicochemical properties [12,16-18]. There are several methods to fluorinate CNTs including fluorinating agent decomposition $[19,20]$, direct fluorination with elemental fluorine, either static or dynamic $[17,21]$, plasma fluorination with fluorocarbons [22,23], and catalytic fluorination [24]. The introduction of fluorine, perfluoroalkyl, and other fluorine-containing moieties into organic compounds can deeply change the physical, chemical, and biological properties of the starting material $[25,26]$. Therefore, CNTs functionalization with fluorinated groups can be considered a suitable technique for transferring the unique features of fluorinated materials like low surface energy as well as high thermal and chemical stability to the carbon-based matrix [27-30]. Perfluoropolyethers (PFPEs) are liquid fluoropolymers embodying supplementary properties such as high gas permeability and high molecular mobility in addition to those of fluorinated materials [27]. PFPE-functionalization can be performed by employing several functional PFPE reagents, but the majority of them contains hydrogenated spacers lack the same thermal and chemical stability of fluoromaterials [28,31,32]. To this aim, PFPE peroxides are advantageous reagents since their thermolysis generates highly reactive perfluorinated radicals, which are able to bond directly with MW-CNTs sidewall without employing detrimental spacers [33]. Therefore, PFPE-functionalization with perfluorinated peroxides can achieve the production of stable superhydrophobic MW-CNTs, which, due to their extraordinary low moisture, find applications in several fields like water management, water-oil separation, self-cleaning, self-lubrication, water repellent surfaces, micro-reactors, and microfluidic systems [34-39].

In the present work, the sidewall functionalization of MW-CNTs with branched and linear PFPE moieties was studied by focusing on the effects ascribable to branching and linearity of PFPE residuals. The covalent linkage of PFPE chains on the MW-CNTs surface was obtained by thermal decomposition of different PFPE peroxides in the presence of MW-CNTs. It was, therefore, expected that the typical properties of fluorinated materials in particular the low surface energy could be transferred to the CNT surface [40]. The static and dynamic contact angle measurements with water were studied in order to characterize the super hydrophobic properties of the functionalized CNT samples. The conductive properties of MW-CNTs and their surface elemental compositions were studied by conductivity measurements and by X-ray photoelectron spectroscopy (XPS), respectively. Morphology changes and surface area variations on MW-CNTs were characterized by scanning electron microscopy (SEM), surface area, and porosity measurements. The thermal stability of the PFPE-functionalized MW-CNTs has been evaluated by thermal gravimetric analysis (TGA). The chemical functionalization with PFPE peroxides was also compared to the physical adsorption of inert PFPE fluids. A specimen of conductive MW-CNTs was also directly fluorinated with elemental fluorine in mild conditions. Its properties were analyzed in order to observe differences and analogies with MW-CNTs functionalized by PFPE chains.

\section{Results}

\subsection{Functionalization Mechanism}

The thermal-induced homolytic cleavage of $\mathrm{O}-\mathrm{O}$ bonds in PFPE peroxides synthesizes highly reactive radicals with PFPE structures, which can directly bond to carbonaceous structures without thermally and chemically detrimental spacers [27,41-43]. In general, the chemical treatments with PFPE peroxides lead to the formation of non-peroxidic PFPE layers covalently linked to the carbon-based surface (see Figure 1). The thermal decomposition of PFPE peroxide generates oxyradicals that produce carbon-centered PFPE radicals by $\beta$-scission reaction. The carbon-centered PFPE radicals participate to the functionalization mechanism in which the graphene layers of MW-CNTs act as radical scavengers performing the chemical linkage of PFPE chains to MW-CNTs sidewalls [34]. At this stage, $\mathrm{sp}^{2}$ carbon atoms change the hybridization to $\mathrm{sp}^{3}$ due to the formation of covalent bonds between PFPE radicals 
and cyclic moieties in graphene sidewalls of MW-CNTs. Carbonyl difluoride, $\mathrm{COF}_{2}$, and acetyl fluoride, $\mathrm{CF}_{3} \mathrm{COF}$, are generated as side-products of the thermolysis of branched PFPE peroxide (see Figure 1a). As shown in Figure $1 b$, if the PFPE peroxide is linear, only $\mathrm{COF}_{2}$ can be produced. The homo-coupling of PFPE radicals competes with MW-CNTs functionalization by forming not-bonded PFPE fluids [27].

The sidewall functionalization of MW-CNTs was performed employing two different PFPE peroxides with similar equivalent molecular weights (EMWs) including a branched PFPE peroxide and a linear PFPE peroxide (see Figure 1). MW-CNTs were suspended in solutions of the corresponding PFPE peroxide dissolved in $\mathrm{CF}_{3} \mathrm{OCFClCF}_{2} \mathrm{Cl}$ and the solvent was then evaporated for depositing molecules of the peroxidic reagent on MW-CNTs surface. The peroxides were thermally decomposed and, therefore, reactive PFPE radicals were produced in the near proximity of MW-CNTs surface. Samples I-BP50 and II-LP50 were prepared by using branched and linear PFPE peroxides, respectively (see Table S1 in the Supplementary Materials).

(a)

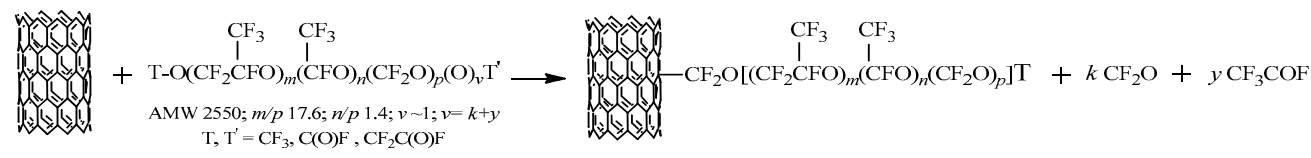

(b)

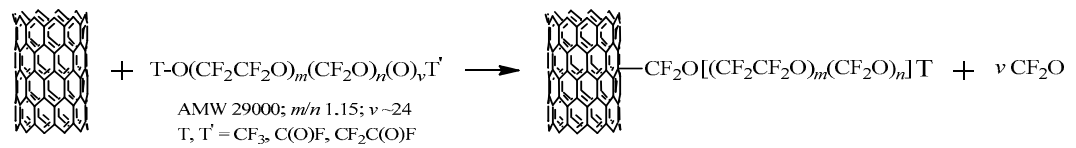

Figure 1. Functionalization reaction of MW-CNTs with branched (a) and linear (b) PFPE peroxides.

The fluorination of MW-CNTs was performed in mild conditions in a below-atmospheric environment of $\mathrm{F}_{2}$ at temperatures ranging from 25 to $80{ }^{\circ} \mathrm{C}$ (sample III-F; Table S2 in the Supplementary Materials). The fluorination of MW-CNTs with $\mathrm{F}_{2}$ is usually associated with a transition from $\mathrm{sp}^{2}$ to $\mathrm{sp}^{3}$ in carbon hybridization by creating covalent $\mathrm{C}-\mathrm{F}$ bonds [44]. Therefore, it was expected that fluorine atoms were covalently bonded to the carbon-based surface and to superficial zone of MW-CNTs. The bulk of the sample was preserved and only surface fluorinated $\mathrm{sp}^{3}$ carbon systems were generated.

The portions of linked, non-linked, and decomposed PFPEs after each functionalization were determined and were expressed as a percentage referred to the corresponding initial load of PFPE peroxide (see Figure 2 and Table S3 in the Supplementary Materials). The PFPEs chains bonded to MW-CNTs were deduced by a mass balance with the other PFPE fractions and resulted equally to approximately $26 \%$ in I-BP50 and 28\% in II-LP50. The portion of linked branched PFPE resulted in roughly similar components to that of linear PFPE because both PFPE peroxides, branched and linear, had comparable EMWs (1275 and $1200 \mathrm{~g} /$ eq, respectively).

Since the carbon-based structure of MW-CNTs is completely stable at the temperatures of the functionalization procedure, it was possible to evaluate the portions of peroxidic PFPE that decomposed to carbonyl difluoride, $\mathrm{CF}_{2} \mathrm{O}$, and acetyl fluoride, $\mathrm{CF}_{3} \mathrm{C}(\mathrm{O}) \mathrm{F}$, by weighting MW-CNTs after the thermal treatment. The $\mathrm{N}_{2}$-inerted reaction environment minimized the production of other by-products ascribable to the presence of oxygen or moisture. The decomposed portions measured during the functionalization of the branched sample, I-BP50, and linear sample, II-LP50, reached around $26 \%$ and $42 \%$, respectively. In pure samples of PFPE peroxides, BP-0 and LP-0, the thermolysis decomposed around $32 \%$ and $41 \%$ of the initial load, respectively. Therefore, the decomposed portions in BP-0 and I-BP50 were lower than those in LP-0 and II-LP50. This difference can be attributed to the presence of a not-peroxidic PFPE content in the starting material of the branched peroxide.

The PFPE radicals that homo-coupled and unachieved the functionalization, formed inert PFPE fluids which were recovered at the end of the treatment and weighted for each sample by evaporating the washing solvents. Referring to the initial load of the corresponding PFPE peroxide, the PFPE fluids 
due to radical homo-coupling were 48\% for I-BP50 and 30\% for II-LP50. The results indicated that the lower the peroxidic content in the polymeric reagent, the higher the relative amount of final PFPE fluids not linked to MW-CNTs sidewalls.

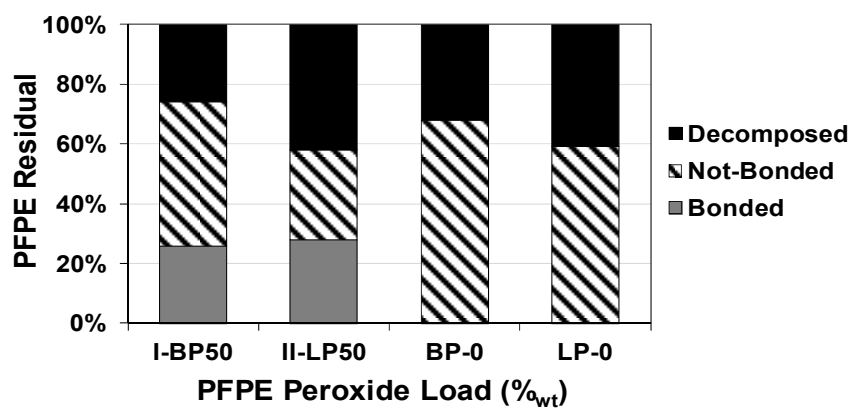

Figure 2. Bonded, non-bonded, and decomposed PFPE residuals after MW-CNTs functionalization compared to decomposition of pure PFPE peroxides.

\subsection{Effect on Wettability}

PFPE-functionalization and fluorination modified the wettability of MW-CNTs from the initial hydrophilic surface properties to the exceeding super-hydrophobicity threshold. SCA measurements on pellets of pure MW-CNTs revealed that the water droplets were adsorbed in a few second (2-4 s) by this carbonaceous matrix because of the porous structure of MW-CNTs bundles. Conversely, the SCA super-hydrophobicity threshold $\left(150^{\circ}\right)$ was exceeded on both branched and linear PFPE-functionalized samples and also on the fluorinated specimen. Moreover, measurements of contact angle hysteresis revealed values below $10^{\circ}$ on all these samples (see Table 1). PFPE peroxides can cover the rough surface of MW-CNTs with highly hydrophobic fluorinated chains [41]. Branched PFPE chains of the functionalized surface have larger encumbrance due to trifluoromethyl groups, $\mathrm{CF}_{3}$, than linear PFPE moieties. Therefore, it was expected that the branched units can generate a higher hydrophobicity than linear units for the same reason that the presence of more electronegative fluorine atoms induces a more marked wettability lowering. The comparison between I-BP50 and II-LP50 confirmed this trend since SCA values of $174^{\circ}$ and $159^{\circ}$ were measured, respectively. The fluorination with elemental fluorine on sample III-F chemically modified the composition of MW-CNTs and a layer of carbon fluorides was generated on the carbon-based surface [45]. Therefore, the synthesis of fluorinated groups on the MW-CNTs surface conferred highly hydrophobic properties to sample III-F and a SCA value around $172^{\circ}$ was recorded, which exceeded the super-hydrophobic threshold (see Table 1).

Table 1. Static and hysteresis contact angle measurements with water, surface composition (at \%), specific surface area, and micropore area of MW-CNTs before and after PFPE-functionalization (I-BP50, II-LP50) and fluorination (III-F).

\begin{tabular}{|c|c|c|c|c|c|c|c|}
\hline \multirow{2}{*}{ Specimen } & \multicolumn{2}{|c|}{ Contact Angle } & \multicolumn{3}{|c|}{$\begin{array}{c}\text { Surface Composition } \\
\text { (at \%) }\end{array}$} & \multirow{2}{*}{$\begin{array}{l}\text { Specific Surface } \\
\text { Area }^{2}\left(\mathrm{~m}^{2} / \mathrm{g}\right)\end{array}$} & \multirow{2}{*}{$\begin{array}{l}\text { Micropore Area } \\
\left(\mathrm{m}^{2} / \mathrm{g}\right)\end{array}$} \\
\hline & Static & Hysteresis & F & $\mathbf{O}$ & $\mathrm{C}$ & & \\
\hline MW-CNTs & n.s. 4 & - & - & 1.3 & 98.7 & 389 & 31 \\
\hline I-BP50 & $174^{\circ}$ & $4.0^{\circ}$ & 9.2 & 2.1 & 88.7 & 231 & 0 \\
\hline II-LP50 & $159^{\circ}$ & $5.3^{\circ}$ & 4.2 & 2.4 & 93.4 & 308 & 0 \\
\hline III-F & $172^{\circ}$ & $4.2^{\circ}$ & 14.2 & 2.0 & 83.8 & 277 & 26 \\
\hline
\end{tabular}

${ }^{1}$ Determined by XPS. ${ }^{2}$ Determined through BET theory. ${ }^{3}$ Determined by $t$-plot method. ${ }^{4}$ not stable. Water droplets are quickly adsorbed (2-4 s) into MW-CNTs pellets.

XPS data showed that the fluorine content on MW-CNTs surface appeared after PFPE-functionalization and fluorination (see Table 1). XPS analysis showed that in samples I-BP50 
and II-LP50, fluorine contents were 9.2 and 4.2 at \%, respectively. The higher fluorine content due to functionalization with branched PFPEs can justify the more marked super-hydrophobicity observed on I-BP50. It is worth noting that, considering that branched and linear PFPE peroxides had similar EMWs, the branched reagent also achieved a higher functionalization degree. The branched PFPE peroxide, which is characterized by a low AMW of $2250 \mathrm{amu}$, functionalized the carbon-based surface and diffused toward the reactive sites into the inner pores of MW-CNTs aggregates. The macromolecular length of linear PFPE peroxide, related to AMW around 29,000 amu, probably hindered its internal diffusion. Due to the high mobility and reactivity (even in mild conditions) of elemental fluorine, the highest fluorine content was measured on sample III-F (14.2 at \%). The oxygen content on the surface of pure MW-CNTs (1.3 at \%) revealed the presence of several oxygenated functions such as alcohols, carbonyls, and carboxyls, which are generally observed. After PFPE-functionalization as well as after fluorination a general increase in oxygen content on MW-CNTs surface was measured. The oxygen contents in samples I-BP50 and II-LP50 were 2.1 and 2.4 at \%, respectively, and were equated stoichiometrically, which is based on the fluorine content due to the linkage of the PFPE chains (see Table 1). On sample III-F, an oxygen increase was observed and it can be ascribed to the presence of oxygen traces or moisture in the reaction environment during the fluorination reaction. The absence of XPS signals due to chlorine atoms in samples I-BP50 and II-LP50 evidenced the complete removal of the solvent $\mathrm{CF}_{3} \mathrm{OCFClCF} 2 \mathrm{Cl}$ by vacuum treatments at high temperature during the functionalization treatment. Thus, the fluorine content was due to PFPE-functionalization on MW-CNTs surface and cannot be attributed to adsorbed solvent.

\subsection{Effect on Morphology}

BET analyses revealed that the morphology of MW-CNTs $\left(389 \mathrm{~m}^{2} / \mathrm{g}\right)$ was separately influenced by functionalization with branched PFPE peroxide or with linear PFPE peroxide as well as by fluorination with elemental fluorine (see Table 1). In sample I-BP50, PFPE-functionalization with branched chains generated a decrease in the surface area to $231 \mathrm{~m}^{2} / \mathrm{g}$. In sample II-LP50, the covalent linkage of linear PFPE chains changed remarkably the hydrophobic properties of MW-CNTs, but the values of the surface area remained high around $308 \mathrm{~m}^{2} / \mathrm{g}$. Direct fluorination with $\mathrm{F}_{2}$ in mild conditions (sample III-F) also decreased MW-CNTs surface area to $277 \mathrm{~m}^{2} / \mathrm{g}$. These data indicate that PFPE-functionalization with branched chains influenced the morphology of native MW-CNTs, which aggregate more than the treatments with linear PFPE peroxide or with $\mathrm{F}_{2}$.

The physical adsorption of PFPE fluids onto the MW-CNTs also generated a variation in the surface properties (see Preparation of Comparative Examples in the Supplementary Materials). After the physisorption of branched and linear PFPE fluids, fluorine contents of 3.8 and 4.7 at \% were observed, respectively, and the surface area decreased to 243 and $229 \mathrm{~m}^{2} / \mathrm{g}$, respectively (see Tables S4 and S5). Contact angle values of $148^{\circ}$ and $170^{\circ}$ were measured, respectively, revealing that the typical hydrophobic properties of the fluorinated chains were conferred to the MW-CNTs surface (see Table S5). However, washings with fluorinated solvent increased the surface area and removed the hydrophobic properties because no chemical linkage of the fluorinated chains occurred in PFPE physisorption (see Table S5). In fact, after washings, no fluorine content on the MW-CNTs surface was detected by XPS analysis as demonstrated by the removal of physisorbed PFPE fluids (see Table S4). Conversely, surface properties like surface composition, surface area, and moisture of samples I-BP50, II-LP50 were permanently stable even after they were washed continuously for $24 \mathrm{~h}$ with pure fluorinated solvent by using a Soxhlet extractor (Tables S4 and S5).

The characterization of pore volume, pore area, and pore size distribution provided quantitative and detailed information about the pore structure of MW-CNTs samples (see Figure 3a,b). It is worth noticing that Barrett-Joyner-Halenda (BJH) method, which is based on the macroscopic Kelvin equation, cannot provide reliable information about the microporous structure. Therefore, only mesoporous and macroporous structures were considered [46]. As shown in Figure 3, volumes and areas of the mesoporous structure in the ranges between 2 to $10 \mathrm{~nm}, 10$ to $25 \mathrm{~nm}$, and 25 to $55 \mathrm{~nm}$ decreased 
significantly after PFPE-functionalization. In particular, volumes and areas of mesopores in these ranges decreased more in sample I-BP50 than in sample II-LP50. This effect can be attributed to the AMW of branched PFPE peroxide, which is significantly lower than that of linear PFPE peroxide. Therefore, due to the relatively short length of its macromolecules, the branched reagent diffused towards the reactive sites located in the internal pores of MW-CNTs aggregates. In the region of the macroporous structure, which include the ranges between 55 to $75 \mathrm{~nm}, 75$ to $100 \mathrm{~nm}$, and more than $100 \mathrm{~nm}$, pore volumes and pore areas slightly decreased in both samples I-BP50 and II-LP50 without evident differences between branched and linear PFPE-functionalization.
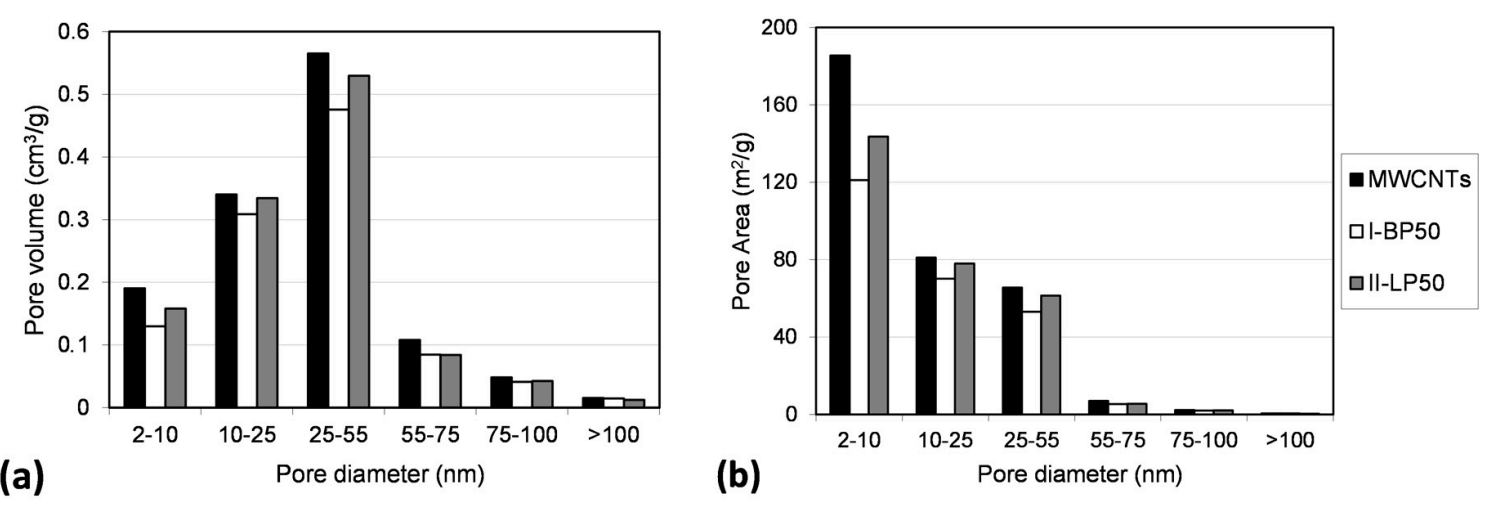

Figure 3. Pore volumes (a) and pore areas (b) of samples before (MW-CNTs) and after PFPEfunctionalization with branched (I-BP50) and linear (II-LP50) peroxides.

The porosity data were normalized by the surface area and the pore distribution was calculated for each sample (see Figure S1). These data revealed that the decrease in the pore structure due to PFPE-functionalization mostly influenced the mesoporosity in the range between 2 to $10 \mathrm{~nm}$. In fact, both branched and linear PFPE peroxides have EMW that fits with PFPE chains, which can enter into MW-CNTs mesopores with this size. The other part of the pore distribution was mainly preserved after PFPE-functionalization, which suggests that PFPE-grafting on MWCNTs surface was almost homogeneous.

MW-CNTs naturally align into "ropes" held together by $\pi$-stacking forces (see Figure $4 \mathrm{a}$ ). The high magnification images of native MW-CNTs showed the aggregation of MW-CNTs in disordered bundles and the remaining interstitial spaces (see Figure $4 \mathrm{~b}$ ). The typical rope-like alignment of MW-CNTs was preserved also on sample I-BP50, in which the layer of branched PFPE covered almost homogeneously MW-CNTs bundles (see Figure 5a) forming only few PFPE aggregates (Figure 5b,c). The polyperoxidic structure of PFPE peroxides allowed the linkage in a row of several connected PFPE chains, which gradually grew the entire PFPEs layers on MW-CNTs surface [47]. Similarly, after treatment with linear PFPE peroxide (see Figure 5d-f) as well as with elemental fluorine (Figure S2 in the Supplementary Materials), the bundled aggregation was overall maintained and avoided MW-CNTs disaggregation.

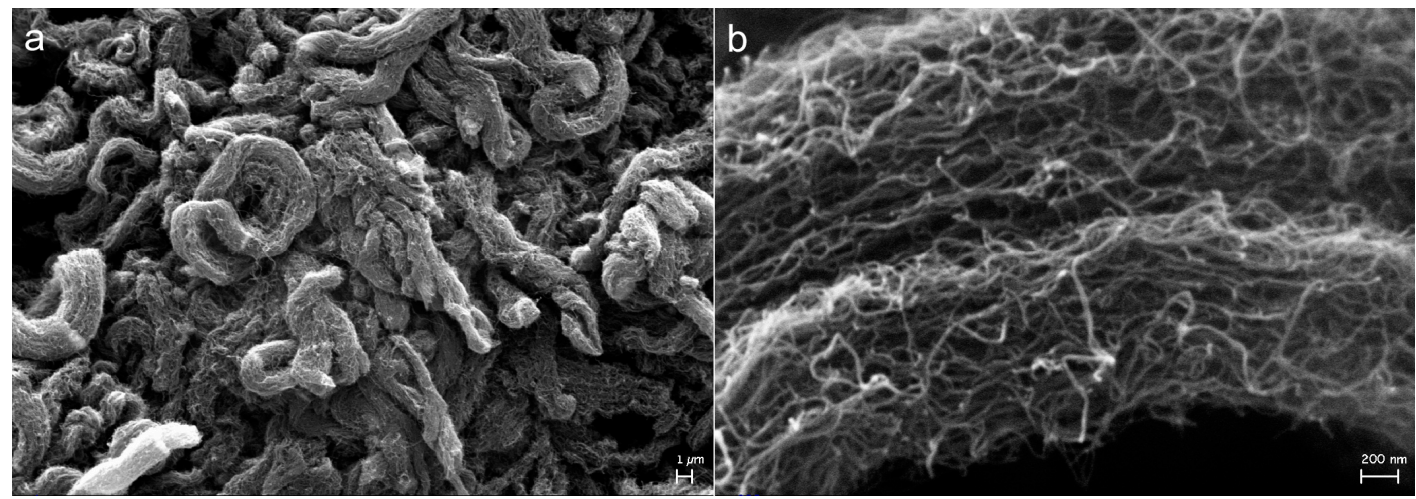

Figure 4. SEM micrographs of native MW-CNTs: 2.5 (a) and $50 \mathrm{kx}(\mathbf{b})$. 

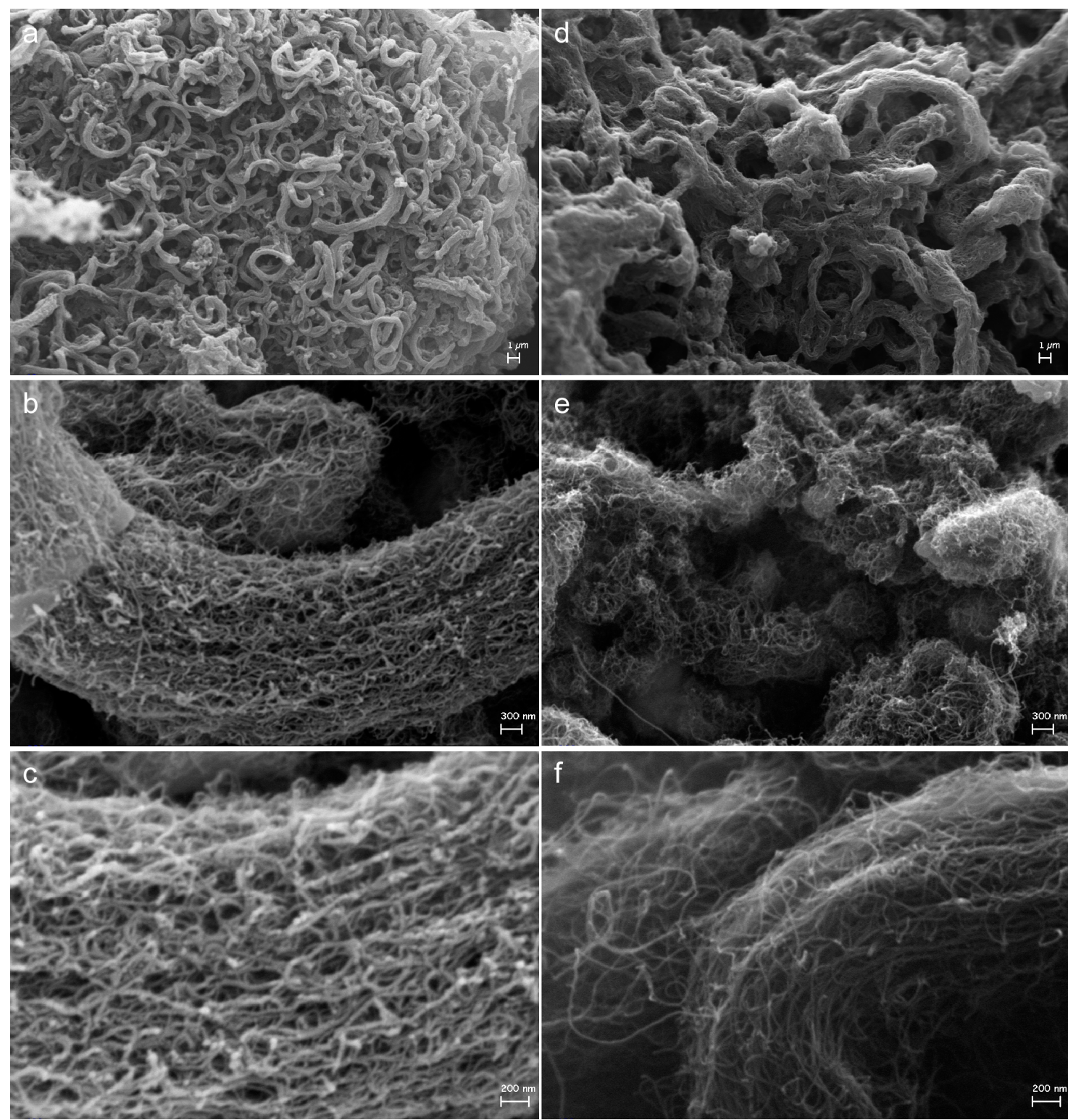

Figure 5. SEM micrographs of MW-CNTs after functionalization with branched (I-BP50) and linear (II-LP50) PFPE peroxides: 2.5 (a,d), 50 (b,e) and $100 \mathrm{kx}(\mathbf{c}, \mathbf{f})$.

\subsection{Effect on Conductive Properties}

MW-CNTs resistivity was evaluated in function of the applied pressure for characterizing the electrical properties before and after PFPE-functionalization as well as after fluorination with $\mathrm{F}_{2}$ (see Figure 6 and, for detailed data, Table S6). The compacting pressure due to the loadings caused resistivity changes ascribable to deformation of the carbon-based porous structure. Additionally, compression increases local contact forces between MW-CNTs for a better contact, which leads to a decrease in the contact resistance between crossing nanotubes [48,49]. For native MW-CNTs (see Figure 6), the electrical resistivity varied in the range $0.1-0.5 \Omega \cdot \mathrm{cm}$. After PFPE-functionalization with branched and linear peroxides, the resistivity values slightly increased in the range of conductive materials (see Figure 6). The ohmic increment was due to the typical non-conductive properties of PFPEs chains endowing MW-CNTs. The electrical resistivity of sample I-BP50 was higher when compared to that of sample II-LP50 because of the higher functionalization degree obtained by the branched reagent. As expected, the highest values of electrical resistivity were measured on $\mathrm{F}_{2}$-fluorinated MW-CNTs 
(III-F): $6.7 \Omega \cdot \mathrm{cm}$ at $0.9 \mathrm{MPa}$ and $1.1 \Omega \cdot \mathrm{cm}$ at $13.6 \mathrm{MPa}$. In addition, $\mathrm{F}_{2}$ fluorination caused the covalent linkage of fluorine atoms to carbon atoms directly on MW-CNTs surface and into the sub-superficial zone of the particles, which changed the $\mathrm{sp}^{2}$ state of the carbon-based layers of the conductive $\pi$-electron system in $\mathrm{sp}^{3}$ hybridization [50]. However, the resistivity values at high applied pressures may suggest that the conductive properties in the bulk of $\mathrm{F}_{2}$-fluorinated MW-CNTs (III-F) were partially preserved.

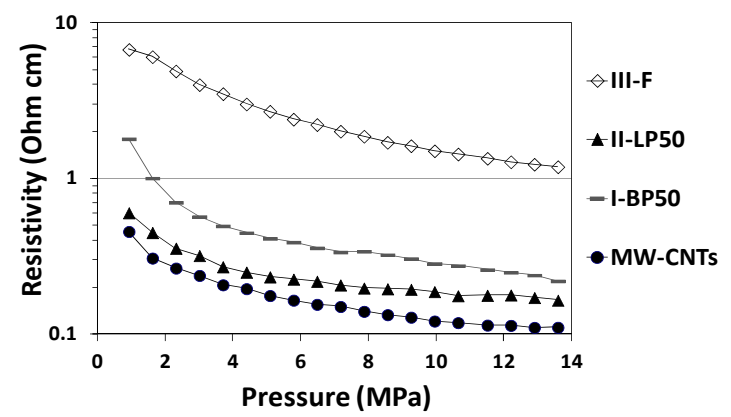

Figure 6. Resistivity (in logarithmic scale) of conductive MW-CNTs at different pressures before (MW-CNTs) and after PFPE-functionalization with branched (I-BP50) and linear (II-LP50) peroxides and after fluorination with $\mathrm{F}_{2}$ (III-F).

\subsection{Effect on Thermal Stability}

The thermal stability of PFPE-functionalized and fluorinated MW-CNTs was evaluated by TGA analysis (see Figure 7). TGA curve of native MW-CNTs revealed that a weak degradation began at around $500{ }^{\circ} \mathrm{C}$ and almost $80 \mathrm{wt} \%$ of the MW-CNTs mass remained stable up to $800{ }^{\circ} \mathrm{C}$ (see Figure $7 \mathrm{a}$ ). MW-CNTs functionalized with branched (I-BP50) and linear (II-LP50) PFPE peroxides (see Figure 7b,c) showed weight losses in two separated steps including the first weight loss ranging from approximately 240-490 ${ }^{\circ} \mathrm{C}$ and it was due to the degradation of PFPE chains grafted on MWCNTs sidewall. A second weight loss started after $500{ }^{\circ} \mathrm{C}$ and it can be ascribed to the degradation of modified parts of carbon nanotubes. For the fluorinated sample III-F, a weight loss of around 12 wt \% occurred between 235 and $500{ }^{\circ} \mathrm{C}$ due to defluorination of MWCNTs (see Figure 7d).

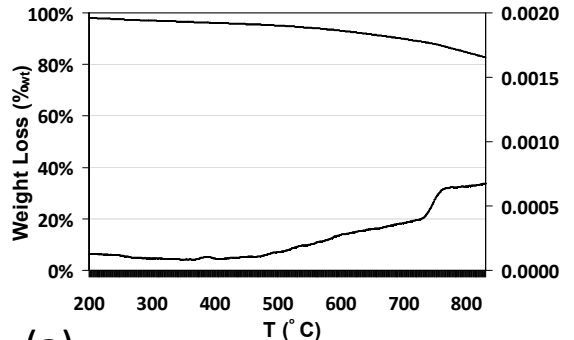

(a)

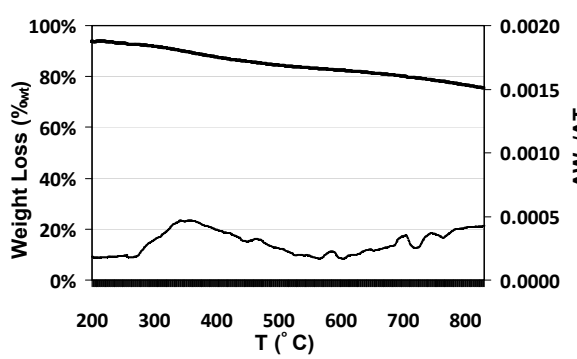

(c)

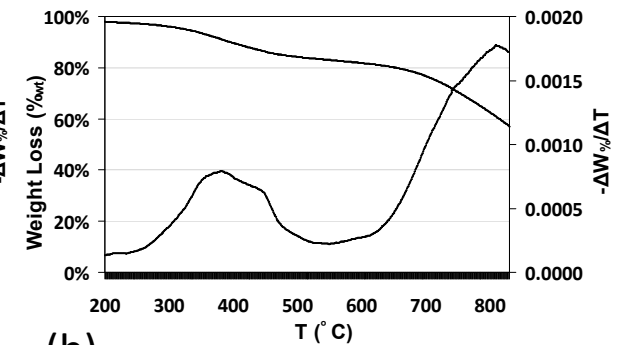

(b)

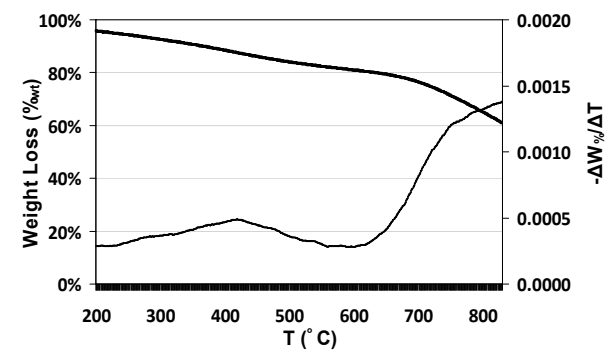

(d)

Figure 7. TGA thermograms of MW-CNTs under $\mathrm{N}_{2}$ before (a) and after PFPE-functionalization with branched (b) and linear (c) peroxides and after fluorination with $\mathrm{F}_{2}(\mathbf{d})$. 


\section{Materials and Methods}

\subsection{Materials}

MW-CNTs (Nanocyl 7000, Sambreville, Belgium) prepared by the supplier through CVD process and were characterized by an average diameter of $9.5 \mathrm{~nm}$ and average length of $1.5 \mu \mathrm{m}$. Branched and linear PFPE peroxides are non-commercial Fomblin ${ }^{\circledR}$ peroxides (Solvay Specialty Polymers, Bollate, Italy). They were synthesized by photooxidative polymerization of tetrafluoroethylene (TFE) and hexafluoropropene (HFP) [45,51] and were kindly provided for this research. Two comparative samples were also prepared by using Fomblin ${ }^{\circledR}$ YHVAC $18 / 8$ and Fomblin ${ }^{\circledR}$ M03, which both do not contain peroxidic moieties. The chemical characteristics of the PFPE compounds are reported below.

- $\quad$ branched PFPE peroxide with general formula TO $\left[\mathrm{CF}_{2} \mathrm{CF}\left(\mathrm{CF}_{3}\right) \mathrm{O}\right]_{\mathrm{m}}\left[\mathrm{CF}\left(\mathrm{CF}_{3}\right) \mathrm{O}\right]_{\mathrm{n}}\left(\mathrm{CF}_{2} \mathrm{O}\right)_{\mathrm{p}}(\mathrm{O})_{\mathrm{v}} \mathrm{T}$ : average molecular weight (AMW) around $2550 \mathrm{amu}$, equivalent molecular weight (EMW) around $1275 \mathrm{~g} / \mathrm{eq}$, ratio between perfluoro-iso-propylene oxide $\left(\mathrm{C}_{3}\right.$, i.e., $\left(\mathrm{CF}_{3}\right) \mathrm{CFCF}_{2} \mathrm{O}$ and $\mathrm{CF}_{2} \mathrm{CF}\left(\mathrm{CF}_{3}\right) \mathrm{O}$ randomly distributed), perfluoro(methyl)methylene oxide $\left(\mathrm{C}_{2}\right.$, i.e., $\left.\mathrm{CF}\left(\mathrm{CF}_{3}\right) \mathrm{O}\right)$ and perfluoromethylene oxide $\left(\mathrm{C}_{1}\right.$, i.e., $\left.\mathrm{CF}_{2} \mathrm{O}\right)$ units $17.6: 1.4: 1$, peroxidic content 0.286 wt \% determined by iodometric titration [52];

- $\quad$ branched PFPE fluid: Fomblin ${ }^{\circledR}$ YHVAC 18/8 by Solvay Specialty Polymers Inc., MWA around $2800 \mathrm{amu}$, ratio between perfluoro-iso-propylene oxide, $\left(\mathrm{C}_{3}\right.$, i.e., $\mathrm{CF}_{2} \mathrm{CF}\left(\mathrm{CF}_{3}\right) \mathrm{O}$ and $\mathrm{CF}_{2} \mathrm{CF}\left(\mathrm{CF}_{3}\right) \mathrm{O}$ randomly distributed), and perfluoromethylene oxide, $\left(\mathrm{C}_{1}\right.$, i.e., $\left.\mathrm{CF}_{2} \mathrm{O}\right)$, units around 15 , no peroxidic moieties along the polymer chain;

- $\quad$ linear PFPE peroxide with general formula $\mathrm{TO}\left(\mathrm{CF}_{2} \mathrm{CF}_{2} \mathrm{O}\right)_{\mathrm{m}}\left(\mathrm{CF}_{2} \mathrm{O}\right)_{\mathrm{n}}(\mathrm{O})_{\mathrm{v}} \mathrm{TO}$ : AMW around 29,000 amu, EMW around $1200 \mathrm{~g} / \mathrm{eq}$, ratio between perfluoroethylene oxide, $\left(\mathrm{C}_{2}\right.$, i.e., $\left.\mathrm{CF}_{2} \mathrm{CF}_{2} \mathrm{O}\right)$, and perfluoromethylene oxide, $\left(\mathrm{C}_{1}\right.$, i.e., $\left.\mathrm{CF}_{2} \mathrm{O}\right)$, units $(m / n)$ around 1.15 , peroxidic content 1.3 wt \%;

- $\quad$ linear PFPE fluid: Fomblin ${ }^{\circledR}$ M03 by Solvay Specialty Polymers Inc. (Woodburn, OR, USA), AMW around $4000 \mathrm{amu}$, ratio between perfluoroethylene oxide, $\left(\mathrm{C}_{2}\right.$, i.e., $\left.\mathrm{CF}_{2} \mathrm{CF}_{2} \mathrm{O}\right)$ and perfluoromethylene oxide, $\left(\mathrm{C}_{1}\right.$, i.e., $\left.\mathrm{CF}_{2} \mathrm{O}\right)$, units $(\mathrm{m} / \mathrm{n})$ around 1 , no peroxidic moieties along the polymer chain $(v=0)$.

$\mathrm{CF}_{3} \mathrm{OCFClCF}_{2} \mathrm{Cl}$ (b.p. $40-41^{\circ} \mathrm{C}$ ) was employed as a solvent because of its absence of reactivity in the presence of PFPE peroxides as well as of PFPE radicals.

Pure elemental fluorine, $\mathrm{F}_{2}$, stored in an appropriate cylinder (8 bar, Solvay Fluor) was employed for direct fluorination of MW-CNTs.

\subsection{PFPE-Functionalization of $M W-C N T s$}

MW-CNTs (6 g) were suspended in a solution of PFPE peroxide $\left(3 \mathrm{~g}\right.$ ) dissolved in $\mathrm{CF}_{3} \mathrm{OCFClCF}_{2} \mathrm{Cl}$ $(150 \mathrm{~mL})$ as fluorinated solvent. The PFPE peroxidic MW-CNTs suspension was homogenized by intense magnetic stirring and sonication $(5 \mathrm{~min})$. Thereafter, the solvent was completely evaporated at $40^{\circ} \mathrm{C}$. PFPE peroxides were thermally decomposed by increasing $\left(5^{\circ} \mathrm{C} / \mathrm{h}\right)$ stepwise the temperature from 150 up to $200^{\circ} \mathrm{C}$, which later remained at $200^{\circ} \mathrm{C}$ for $2 \mathrm{~h}$. At the end of the thermal treatment, the residue of functionalized MW-CNTs was recovered, filtered on a PTFE-membrane with pore size of $0.45 \mu \mathrm{m}$ (Sartorius Stedim Biotech, Göttingen, Germany) and washed three times with $\mathrm{CF}_{3} \mathrm{OCFClCF}_{2} \mathrm{Cl}$ $(150 \mathrm{~mL})$ and three times with deionized water $(150 \mathrm{~mL})$. PFPE-functionalized MW-CNTs were finally dried under vacuum $(0.01 \mathrm{mmHg})$ at $200{ }^{\circ} \mathrm{C}$ for $24 \mathrm{~h}$. Samples I-BP50 and II-LP50 were prepared by using branched and linear PFPE peroxide, respectively (see Table S1 in the Supplementary Materials).

PFPE-functionalization was compared to direct fluorination of MW-CNTs with $\mathrm{F}_{2}$. A fluorine pressure of around $100 \mathrm{mbar}$ was introduced in the reactor for $15 \mathrm{~min}$ and, thereafter, the reactor was evacuated forcing all the gases to a soda lime trap. This procedure was repeated eight times at room temperature and two times at $80^{\circ} \mathrm{C}$. The conditions of the fluorination reaction (sample III-F) were reported in Table S2 in the Supplementary Materials. 
Each sample of MW-CNTs was weighted before and after the thermal treatment in order to determine the portion of peroxidic PFPE that decomposed to carbonyl difluoride, $\mathrm{CF}_{2} \mathrm{O}$, and acetyl fluoride, $\mathrm{CF}_{3} \mathrm{C}(\mathrm{O}) \mathrm{F}$, during the thermolysis, which assumed a complete stability of $\mathrm{CNTs}$ matrix until $200{ }^{\circ} \mathrm{C}$. The portion of PFPE that homo-coupled, shirking MW-CNTs functionalization, was evaluated by weighting the PFPE residue, which was obtained by evaporating the washing solvents. The portion of linked PFPE was obtained as the difference. The portions of linked, non-linked, and decomposed PFPEs were expressed as percentage referred to the corresponding initial load of PFPE peroxide.

\subsection{Characterizations}

DSA100 Series Instrument (Kruss, Hamnburg, Germany) equipped with DSA1 software (version 1.29.1.1, Kruss, Hamnburg, Germany) was employed for contact angle measurements. Pellets of MW-CNTs samples (5-20 mg) were prepared by press molding at room temperature under a load of $7000 \mathrm{~kg} / \mathrm{cm}^{2}$ for a few minutes (3-5 min). Water droplets were deposited on MW-CNTs surface for SCA measurements. Drop volumes for advancing and receding contact angles were in the range from 8 to $20 \mu \mathrm{L}$. The hysteresis was calculated by subtracting the measured advancing contact angles with the measured receding contact angles.

An M-probe spectrometer (Surface Science Instrument, SSI, Mountain View, CA, USA) was used for X-ray photoelectron spectroscopy. The spectra were obtained by a monochromatic X-ray emission of $\mathrm{A} 1 \mathrm{~K} \alpha$ radiation $(1486.6 \mathrm{eV})$. A spot size of $200 \times 750 \mu \mathrm{m}$ and pass energy of $29 \mathrm{eV}$ were used. $1 \mathrm{~s}$ level hydrocarbon-contaminant carbon was taken as the internal reference at $284.6 \mathrm{eV}$.

The specific surface areas were measured by $\mathrm{N}_{2}$ adsorption and calculated on the basis of Brunauer-Emmett-Teller (BET) theory. The $\mathrm{N}_{2}$ adsorption allowed for the determination of the total microporous surface area by means of the $t$-plot method. Pore volumes and pore areas were determined as a function of the pore diameter in the mesoporous and macroporous ranges by using the $\mathrm{BJH}$ method. Before analysis, sample were pre-treated under vacuum $(0.1 \mathrm{mmHg})$ at $130^{\circ} \mathrm{C}$ for $2.5 \mathrm{~h}$. A Micromeritics TriStar II 3020, managed by the software TriStar II 3020 V1.03 (Norcross, GA, USA), was used for BET analysis and porosimetry.

Scanning electron microscope (Zeiss EVO-50, Zeiss, Thornwood, NY, USA), working distance $8.0 \mathrm{~mm}$, beam current $100 \mathrm{pA}$, acceleration voltage $20.00 \mathrm{kV}$ ) was employed to analyze the morphology of MW-CNTs samples. Microscopy was performed on bare samples without deposition of a conductive layer.

The electrical resistivity measurements at different applied pressures (0.9-13.6 MPa) were performed by using a tailor-made apparatus assembled according to literature description [53]. The resistance $(R)$ values were measured by using an AOIP OM21 Micro-ohmmeter (AOIP, Paris, France) and were converted in resistivity ( $\rho$ ) by applying Equation (1).

$$
\rho=\frac{R \cdot \pi \cdot d^{2}}{4 l}=\frac{1}{\mathcal{\kappa}}
$$

in which $d$ is the diameter of apparatus tube, $l$ is the distance between the two pistons of the apparatus, and $k$ is the specific conductivity. Measurements were repeated three times on each MW-CNTs sample at the same pressures in order to decrease the experimental error of the test.

The thermogravimetric analyses were performed with a THASS TGA XP-10 (Thass, Friedberg, Germany)) analyzer. The samples (approximately $10 \mathrm{mg}$ ) were heated from 200 to $830^{\circ} \mathrm{C}$ at a rate of $10^{\circ} /$ min under $\mathrm{N}_{2}$ flow.

\section{Conclusions}

PFPE-functionalization of MW-CNTs was obtained by thermal decomposition of branched and linear PFPE peroxides. The linkage of PFPEs chains conferred super-hydrophobic properties to MW-CNTs surface, which was proven by contact angle measurements. The comparison between branched and linear PFPE-functionalization revealed that the branched reagent achieved a higher 
functionalization degree than the linear reagent. Porosimetry showed that branched PFPE peroxide reached the reactive sites located in the internal MW-CNTs mesopores due to the relatively short length of its molecules while linear PFPE-functionalization mostly preserved MW-CNTs mesomorphology. Macropores were partially influenced by PFPE-functionalization without evident differences related to the use of branched or linear reagents. Moreover, the marked moisture decreasing in branched PFPE-functionalized MW-CNTs is also due to the presence of more electronegative fluorine atoms due to trifluoromethyl groups, $\mathrm{CF}_{3}$. The SEM images indicated that MW-CNTs maintained rope-like structures after functionalization and disaggregation was not observed. PFPE-functionalized MW-CNTs remained electrically conductive even if their moisture varied significantly. In summary, PFPE-functionalization of MW-CNTs can be employed for a controlled modification of surface and morphological properties of carbon nanotubes without detriment to their conductivity.

Supplementary Materials: The following are available online at http:/ /www.mdpi.com/2079-4991/8/3/176/s1, Table S1: Experimental conditions of MW-CNTs functionalization with branched and linear PFPE peroxides, Table S2: Experimental conditions of MW-CNTs fluorination with $\mathrm{F}_{2}$, Table S3: Weight evaluations for determination of linked, non-linked and decomposed portions of PFPEs in MW-CNTs functionalization with PFPE peroxides, Preparation of Comparative Examples-Physisorption of linear and branched PFPE Fluids on MW-CNTs, Table S4: Surface composition (at \%) by XPS analysis referred to the comparative example, Table S5: Contact angle with water and BET surface area measurements referred to the comparative example, Table S6: Electrical resistivity at different pressures of MW-CNTs before and after PFPE-functionalization with branched and linear PFPE peroxides and after fluorination with $\mathrm{F}_{2}$, Figure S1: Normalized pore volumes (a) and pore areas (b) of the samples before (MW-CNTs) and after PFPE-functionalization with branched and linear peroxides (I-BP50 and II-LP50), Figure S2: SEM micrographs of fluorinated MW-CNTs (III-F): 2.5 (a) and 100 kx (b).

Acknowledgments: This paper is dedicated to the memory of our wonderful colleague, the microscopist Dario Picenoni, who recently passed away.

Author Contributions: Maurizio Sansotera and Walter Navarrini conceived and designed the experiments. Sadaf Talaeemashhadi performed the experiments. Cristian Gambarotti, Carlo Pirola, Mariangela Longhi and Marco A. Ortenzi analyzed the data. Walter Navarrini and Claudia L. Bianchi contributed reagents/materials/analysis tools Maurizio Sansotera wrote the paper.

Conflicts of Interest: The authors declare no conflict of interest.

\section{References}

1. Iijima, S. Helical microtubules of graphitic carbon. Nature 1991, 354, 56-58. [CrossRef]

2. Ebbesen, T.W. Carbon Nanotubes: Preparation and Properties, 1st ed.; CRC Press: Boca Raton, FL, USA, 1997; pp. 191-249.

3. Engels, V.; Geng, J.; Jones, G.M.; Elliott, J.A.; Wheatley, A.E.H.; Boss, S.R. Cobalt catalyzed carbon nanotube growth on graphitic paper supports. Curr. Nanosci. 2011, 7, 315-322. [CrossRef]

4. Ebbesen, T.W.; Lezec, H.J.; Hiura, H.; Bennett, J.M.; Ghaemi, H.F.; Thio, T. Electrical conductivity of individual carbon nanotubes. Nature 1996, 382, 54-56. [CrossRef]

5. De Heer, W.A.; Bacsa, W.S.; Chatelain, A.; Gergin, T.; Humphrey-Baker, R.; Forro, L.; Ugarte, D. Aligned carbon nanotube films: Production and optical and electronic properties. Science 1995, 268, 845-847. [CrossRef] [PubMed]

6. Delaney, P.; Choi, H.J.; Ihm, J.; Cohen, M.L. Broken symmetry and pseudogaps in ropes of carbon nanotubes. Nature 1998, 391, 466-468. [CrossRef]

7. Orinakova, R.; Orinak, A. Recent applications of carbon nanotubes in hydrogen production and storage. Fuel 2011, 90, 3123-3140. [CrossRef]

8. Lee, J.Y.; An, K.H.; Heo, J.K.; Lee, Y.H. Fabrication of supercapacitor electrodes using fluorinated single-walled carbon nanotubes. J. Phys. Chem. 2003, 107, 8812-8815. [CrossRef]

9. Endo, M.; Strano, M.S.; Ajayan, P.M. Potential applications of carbon nanotubes. In Carbon Nanotubes: Advanced Topics in the Synthesis, Structure, Properties and Applications (Topics in Applied Physics); Jorio, A., Dresselhaus, G., Dresselhaus, M.S., Eds.; Springer: New York, NY, USA, 2008; pp. 13-61.

10. Holzinger, M.; Vostrowsky, O.; Hirsch, A.; Hennrich, F.; Kappes, M.; Weiss, R.; Jellen, F. Sidewall functionalization of carbon nanotubes. Angew. Chem. Int. Ed. 2001, 40, 4002-4005. [CrossRef] 
11. Martínez-Hernández, A.L.; Velasco-Santos, C.; Castaño, V.M. Carbon nanotubes composites: Processing, grafting and mechanical and thermal properties. Curr. Nanosci. 2010, 6, 12-39. [CrossRef]

12. Khabashesku, V.N.; Billups, W.E.; Margrave, J.L. Fluorination of single-wall carbon nanotubes and subsequent derivatization reactions. Acc. Chem. Res. 2002, 35, 1087-1095. [CrossRef] [PubMed]

13. Hu, H.; Zhao, B.; Hamon, M.A.; Kamaras, K.; Itkis, M.E.; Haddon, R.C. Sidewall functionalization of single-walled carbon nanotubes by addition of dichlorocarbene. J. Am. Chem. Soc. 2003, 125, 14893-14900. [CrossRef] [PubMed]

14. Georgakilas, V.; Kordatos, K.; Prato, M.; Guldi, D.M.; Holzinger, M.; Hirsch, A. Organic functionalization of carbon nanotubes. J. Am. Chem. Soc. 2002, 124, 760-761. [CrossRef] [PubMed]

15. Ying, Y.; Saini, R.K.; Liang, F.; Sadana, A.K.; Billups, W.E. Functionalization of carbon nanotubes by free radicals. Org. Lett. 2003, 5, 1471-1473. [CrossRef] [PubMed]

16. Tressaud, A.; Shirasaki, T.; Nanse, G.; Papirer, E. Fluorinated carbon blacks: Influence of the morphology of the starting material on the fluorination mechanism. Carbon 2002, 40, 217-220. [CrossRef]

17. Lee, Y.S.; Cho, T.H.; Lee, B.K.; Rho, J.S.; An, K.H.; Lee, Y.H. Surface properties of fluorinated single-walled carbon nanotubes. J. Fluor. Chem. 2003, 120, 99-104. [CrossRef]

18. Chamssedine, F.; Guérin, K.; Dubois, M.; Disa, E.; Petit, E.; Fawal, Z.E.; Hamwi, A. Fluorination of single walled carbon nanotubes at low temperature: Towards the reversible fluorine storage into carbon nanotubes. J. Fluor. Chem. 2011, 132, 1072-1078. [CrossRef]

19. Zhang, W.; Guérin, K.; Dubois, M.; Fawal, Z.E.; Ivanov, D.A.; Vidal, L.; Hamwi, A. Carbon nanofibres fluorinated using $\mathrm{TbF}_{4}$ as fluorinating agent. Part 1: Structural properties. Carbon 2008, 46, 1010-1016. [CrossRef]

20. Umemoto, T. Recent advances in perfluoroalkylation methodology. In Fluorine-Containing Synthons; Soloshonok, V.A., Ed.; American Chemical Society: Denver, CO, USA, 2005; pp. 2-15.

21. Wang, Y.Q.; Sherwood, P.M.A. Studies of carbon nanotubes and fluorinated nanotubes by X-ray and ultraviolet photoelectron spectroscopy. Chem. Mater. 2004, 16, 5427-5436. [CrossRef]

22. Valentini, L.; Puglia, D.; Carniato, F.; Boccaleri, E.; Marchese, L.; Kenny, J.M. Use of plasma fluorinated single-walled carbon nanotubes for the preparation of nanocomposites with oxymatrix. Compos. Sci. Technol. 2008, 68, 1008-1014. [CrossRef]

23. Tressaud, A.; Durand, E.; Labrugère, C. Surface modification of several carbon-based materials: Comparison between $\mathrm{CF}_{4} \mathrm{rf}$ plasma and direct $\mathrm{F}_{2}$-gas fluorination routes. J. Fluor. Chem. 2004, 125, 1639-1648. [CrossRef]

24. Guerin, K.; Dubois, M.; Houdayer, A.; Hamwi, A. Applicative performances of fluorinated carbons through fluorination routes: A review. J. Fluor. Chem. 2012, 134, 11-17. [CrossRef]

25. Hudlicky, M.; Pavlath, A.E. Chemistry of Organic Fluorine Chemistry II: A Critical Review (ACS Monograph 187); American Chemical Society: Washington, DC, USA, 1995.

26. Sansotera, M.; Navarrini, W.; Gola, M.; Dotelli, G.; Gallo Stampino, P.; Bianchi, C.L. Conductivity and superhydrophobic effect on PFPE-modified porous carbonaceous materials. Int. J. Hydrogen Energy 2012, 37, 6277-6284. [CrossRef]

27. Sianesi, D.; Marchionni, G.; Pasquale, R.J.D. Perfluoropolyethers (PFPEs) from perfluoroolefin photooxidation. In Organo Fluorine Chemistry: Principles and Commercial Applications (Topics in Applied Chemistry); Banks, R.E., Smart, B.E., Tatlow, J.C., Eds.; Plenum Press: New York, NY, USA, 1994; pp. 431-461.

28. Persico, F.; Sansotera, M.; Diamanti, M.V.; Magagnin, L.; Venturini, F.; Navarrini, W. Effect of amorphous fluorinated coatings on photocatalytic properties of anodized titanium surfaces. Thin Solid Films 2013, 545, 210-216. [CrossRef]

29. Avataneo, M.; Navarrini, W.; De Patto, U.; Marchionni, G. Novel perfluoropolyethers containing 2,2,4-trifluoro-5-trifluoromethoxy-1,3-dioxole blocks: Synthesis and characterization. J. Fluor. Chem. 2009, 130, 933-937. [CrossRef]

30. Navarrini, W.; Diamanti, M.V.; Sansotera, M.; Persico, F.; Menghua, W.; Magagnin, L.; Radice, S. UV-resistant amorphous fluorinated coating for anodized titanium surfaces. Prog. Org. Coat. 2012, 74, 794-800. [CrossRef]

31. Demir, T.; Wei, L.; Nitta, N.; Yushin, G.; Brown, P.J.; Luzinov, I. Toward a long-chain perfluoroalkyl replacement: Water and oil repellency of polyethylene terephthalate (PET) films modified with perfluoropolyether-based polyesters. ACS Appl. Mater. Interfaces 2017, 9, 24318-24330. [CrossRef] [PubMed]

32. Walters, K.B.; Schwark, D.W.; Hirt, D.E. Surface characterization of linear low-density polyethylene films modified with fluorinated additives. Langmuir 2003, 19, 5851-5860. [CrossRef] 
33. Guarda, P.A.; Barchiesi, E.; Fontana, G.; Petricci, S.; Pianca, M.; Marchionni, G. Peroxidic perfluoropolyether from tetrafluoroethylene oxidation: Micro structural analysis by NMR spectroscopy and mechaniostic considerations. J. Fluor. Chem. 2005, 126, 141-153. [CrossRef]

34. Yang, Y.; Li, X.; Zheng, X.; Chen, Z.; Zhou, Q.; Chen, Y. 3D-printed biomimetic super-hydrophobic structure for microdroplet manipulation and oil/water separation. Adv. Mater. 2018, 30, 1704912. [CrossRef] [PubMed]

35. Sansotera, M.; Gola, M.; Navarrini, W. Perfluoropolyether-functionalized carbon-based materials and their applications. In New Fluorinated Carbons: Fundamentals and Applications; Boltalina, O.V., Nakajima, T., Tressaud, A., Eds.; Elsevier: Amsterdam, The Netherlands, 2017; pp. 361-392.

36. Sansotera, M.; Gola, M.; Dotelli, G.; Navarrini, W. The role of perfluoropolyethers in the development of polymeric proton exchange membrane fuel cells. In Fluorinated Polymers Volume 2: Applications; Ameduri, B., Sawada, H., Eds.; The Royal Society of Chemistry: Cambridge, UK, 2017; pp. 158-178.

37. Zhao, F.; Franz, S.; Vicenzo, A.; Cavallotti, P.L.; Sansotera, M.; Navarrini, W. Electrodeposition of nanostructured columnar cobalt for self-lubricant coatings. Electrochim. Acta 2011, 56, 9644-9651. [CrossRef]

38. Yan, K.-K.; Jiao, L.; Lin, S.; Ji, X.; Lu, Y.; Zhang, L. Superhydrophobic electrospun nanofiber membrane coated by carbon nanotubes network for membrane distillation. Desalination 2018, 437, 26-33. [CrossRef]

39. Wang, C.-F.; Hung, S.-W.; Kuo, S.-W.; Chang, C.-J. Combining hierarchical surface roughness with fluorinated surface chemistry to preserve superhydrophobicity after organic contamination. Appl. Surf. Sci. 2014, 320, 658-663. [CrossRef]

40. Hattori, Y.; Watanabe, Y.; Kawasaki, S.; Okino, F.; Pradhan, B.K.; Kyotani, T.; Tomita, A.; Touhara, H. Carbon-alloying of the rear surfaces of nanotubes by direct fluorination. Carbon 1999, 37, 1033-1038. [CrossRef]

41. Li, G.; Kaneko, K.; Ozeki, S.; Okino, F.; Touhara, H. Water rejective nature of fluorinated microporous carbon fibers. Langmuir 1995, 11, 716-717. [CrossRef]

42. Sansotera, M.; Navarrini, W.; Gola, M.; Bianchi, C.L.; Wormald, P.; Famulari, A.; Avataneo, M. Peroxidic perfluoropolyether for the covalent binding of perfluoropolyether chains on carbon black surface. J. Fluor. Chem. 2012, 132, 1254-1261. [CrossRef]

43. Sansotera, M.; Bianchi, C.L.; Lecardi, G.; Marchionni, G.; Metrangolo, P.; Resnati, G.; Navarrini, W. Highly hydrophobic carbon black obtained by covalent linkage of perfluorocarbon and perfluoropolyether chains on the carbon surface. Chem. Mater. 2009, 21, 4498-4504. [CrossRef]

44. Hirsch, G.; Vostrowsky, O. Functionalization of carbon nanotubes. In Functional Organic Materials: Syntheses, Strategies and Applications; Muller, T.J.J., Bunz, U.H.F., Eds.; Wiley-VCH Verlag GmbH \& Co: Weinheim, Germany, 2007; pp. 1-57.

45. Galimberti, M.; Barchiesi, E.; Navarrini, W. $\alpha$-Branched perfluorodiacyl peroxides: Preparation and characterization. J. Fluor. Chem. 2005, 126, 587-593. [CrossRef]

46. Thommes, M.; Kohn, R.; Froba, M. Systematic sorption studies on surface and pore size characteristics of different MCM-48 silica materials. In Studies in Surface Science and Catalysis, Vol. 128-Characterisation of Porous Solids V, Proceedings of the 5th International Symposium on the Characterisation of Porous Solids (COPS-V), Heidelberg, Germany, 30 May-2 June 1999; Unger, K.K., Kreysa, G., Baselt, J.P., Eds.; Elsevier: Amsterdam, The Netherlands, 2000; pp. 259-268.

47. Talaeemashhadi, S.; Sansotera, M.; Gambarotti, C.; Famulari, A.; Bianchi, C.L.; Guarda, P.A.; Navarrini, W. Functionalization of multi-walled carbon nanotubes with perfluoropolyether peroxide to produce superhydrophobic properties. Carbon 2013, 59, 150-159. [CrossRef]

48. Poquillon, D.; Viguier, B.; Andrieu, E. Experimental data about mechanical behavior during compression tests for various matted fibres. J. Mater. Sci. 2005, 40, 5963-5970. [CrossRef]

49. Slobodian, P.; Riha, P.; Olejnik, R.; Saha, P. Electromechanical properties of carbon nanotube networks under compression. Meas. Sci. Technol. 2011, 22, 1-7. [CrossRef]

50. Touhara, H.; Okino, F. Property control of carbon materials by fluorination. Carbon 2000, 38, $241-267$. [CrossRef]

51. Sansotera, M.; Navarrini, W.; Magagnin, L.; Bianchi, C.L.; Sanguineti, A.; Metrangolo, P.; Resnati, G. Hydrophobic carbonaceous materials obtained by covalent bonding of perfluorocarbon and perfluoropolyether chains. J. Mater. Chem. 2010, 20, 8607-8616. [CrossRef] 
52. Navarrini, W.; Bianchi, C.L.; Magagnin, L.; Nobili, L.; Carignano, G.; Metrangolo, P.; Resnati, G.; Sansotera, M. Low surface energy coatings covalently bonded on diamond-like carbon films. Diam. Relat. Mater. 2010, 19, 336-341. [CrossRef]

53. Sanchez-Gonzalez, J.; Macias-Garcia, A.; Alexandre-Franco, M.F.; Gomez-Serrano, V. Electrical conductivity of carbon blacks under compression. Carbon 2005, 43, 741-747. [CrossRef] 